\title{
SOBRE O VERIFICACIONISMO \\ E RESTRIÇÕES AO PRINCÍPIO DO TERCEIRO EXCLUIIDO
}

Marcos Silva ${ }^{1}$

\begin{abstract}
RESUMO
O presente artigo mostra como relacionar o problema do Wittgenstein Intermediário em expressar relações conceituais entre cores a alguns problemas com suas observações verificacionistas. Discute-se também alguns problemas concernentes ao princípio do terceiro excluído (PTE) no contexto das Satzsysteme e do verificacionismo em algumas discussões do Círculo de Viena. Para tanto, investiga-se a relação entre inferencialismo e verificacionismo. Lidar-se-á com o assim chamado problema da exclusão de cores e seu impacto no meio notacional tractariano, mas mostrando que tabelas de verdade podem expressar relações de contrariedade, subcontrariedade e contraditoriedade a partir de mutilações sistemáticas de suas linhas. Também se examina como e por que nos sistemas fenomenológicos PTE parece não valer.
\end{abstract}

Palavras-chave: Verificacionismo. Expressivismo. Inferencialismo. Contradição. Contrariedade. Wittgenstein Intermediário.

\begin{abstract}
Here I show how to relate Middle Wittgenstein's problem with the expression of conceptual relations among colors to some problems to his verificationist remarks. Some problems concerning the Principle of Excluded Middle (PEM) are also discussed in the context of Satzsysteme and verificationism in the the discussions with the Vienna Circle. For that, this work investigates the so-called color exclusion problem and its impact on the Tractarian notational means, however, showing that truth-table notation can express relations of contrariety, subcontrary and contradictoriness through systematic mutilations of its lines. I also examine how and why (PEM) seems to be not valid among phenomenological systems.
\end{abstract}

Keywords: Verificationism. Expressivism. Inferentialism. Contradiction. Contrariety. Middle Wittgenstein.

\footnotetext{
${ }^{1}$ Professor associado do Departamento de Filosofia da Universidade Federal de Alagoas e Pesquisador de Produtividade nível 2 do CNPq. marcossilvarj@gmail.com.
} 


\section{Introdução}

A interpretação, origem, pressupostos e consequências do princípio de verificação no contexto das discussões do Círculo de Viena são problemáticos. Especialmente, há explicações diversas para a invenção ou aceitação do verificacionismo no início do período intermediário da Filosofia de Wittgenstein. Por um lado, Max Black (1986) e Alberto Coffa (1991) defendem que o verificacionismo de vida curta na filosofia de Wittgenstein teria uma importância crucial para seu desenvolvimento filosófico especialmente na visão que associa significado a uso. Por outro lado, Medina (2001) acredita que é a noção de significado como uso que motiva o verificacionismo do Wittgenstein Intermediário. Ainda há uma leitura que articula intimamente o princípio de verificação e o empirismo, como Ayer (1936) ou Carnap (1963). Como mostra Engelmann (2018), os membros do Círculo de Viena pensaram que o princípio teria sido fundado em uma ideia positivista, a saber, que toda proposição deveria ser traduzida em termos de dados imediatos do sentido ou experiência.

Diferentemente dos membros do Círculo de Viena, Wittgenstein não concebia a verificação de uma maneira negativa, como uma ferramenta para eliminar pseudoproblemas. Segundo Engelmann (2018), para o Wittgenstein intermediário, verificação constituiria o método (ou conjunto de métodos) para determinar o sentido de uma proposição. Como consequência, métodos de verificação deveriam tornar explícitas as regras da gramática pertencentes a inúmeros espaços fenomenológicos (especialmente, o de cores).

O presente artigo mostra como relacionar o problema do Wittgenstein Intermediário em expressar relações conceituais entre cores a alguns problemas com suas observações verificacionistas que emergem no contexto fenomenológico das suas Satzsysteme. Apresenta-se também alguns problemas concernentes ao princípio do terceiro excluído (PTE) no contexto das Satzsysteme e do verificacionismo em algumas discussões do Círculo de Viena. Para tanto, investiga-se na primeira seção a relação entre inferencialismo e verificacionismo. A seção dois lidará com o assim chamado problema da exclusão de cores e seu impacto no meio notacional tractariano, mostrando que tabelas de verdade podem expressar relações de contrariedade, sub- 
contrariedade e contraditoriedade a partir de mutilações sistemáticas de suas linhas. A terceira seção examina como e por que nos sistemas fenomenológicos PTE parece não valer.

\section{Sobre o inferencialismo do Wittgenstein Intermediário}

Engelmann $(2013,2014,2017,2018)$ explica as noções fenomenológicas e verificacionistas em torno de 1929-1930, no assim chamado começo do período intermediário de Wittgenstein, sob a luz de sua filosofia pregressa. Segundo Engelmann, Medina (2001) está correto em sua avaliação que relaciona o verificacionismo de Wittgenstein Intermediário com formas de inferencialismo.

Embora concorde com o caráter 'inferencialista' envolvido na aceitação do critério de verificação, Engelmann (2014) critica Medina ao afirmar que:

Sua explicação da introdução do mesmo não é adequada. Pensa que o critério é introduzido para o estabelecimento da "identidade de sistemas". Para tanto, supõe que um sistema matemático funciona do mesmo modo que um sistema de proposições fenomenológicas. Essa suposição confunde o uso matemático e o uso fenomenológico do critério. Em matemática, um sistema e seus métodos de prova determinam completamente o sentido de "proposições" e o significado de sinais (PB $\S \S ~ 49-5)$. Contudo, esse não é o caso de proposições contingentes e seus sinais descritivos, uma vez que nelas a referência ao mundo é essencial (a referência fenomênica de palavras descritivas é parte de seu significado). A questão a respeito da identidade de um sistema matemático tampouco se coloca, uma vez que "não podemos procurar por um sistema" (PB §5). Outro equívoco de Medina, penso, é não perceber que o contexto das $\mathrm{PB}$ não é o mesmo das aulas de 32. (p. 14)

Com efeito, Medina confunde sistemas fenomenológicos com um sistema matemático e equivocadamente não percebe que a Philosophische Bemerkungen $\left(\mathrm{PB}^{2}\right)$ não está no mesmo contexto filosófico das Lectures de 1932. Este último equívoco de Medina parece motivar sua interpretação anacrônica de que o princípio da verificação apareceria como consequência de uma semântica já associada ao uso, uma vez que, em 1932, Wittgenstein

\footnotetext{
${ }^{2}$ Todos os números decimais presentes neste texto vêm da numeração das passagens do Tractatus. As traduções tanto do alemão quanto do inglês são todas de minha responsabilidade. Uso aqui PB para 'Philosophische Bemerkungen', SRLF para 'Some Remarks of Logical Forms', e WWK para 'Wittgenstein und der Wiener Kreis'.
} 
já discutia, assim como em sua filosofia madura, o papel semântico do uso determinando sentido proposicional de expressões linguísticas. Entretanto, a discussão explícita sobre o papel do uso na determinação do sentido proposicional não aparece no contexto do começo da fase intermediária de Wittgenstein (Hacker, 1986).

Em contraste com Medina, Engelmann (2018) defende que

[...] the understanding of methods of verification makes explicit rules of 'grammar' related to phenomenological spaces that show what is meant by a sentence and how it is understood. Thus, I call Wittgenstein's kind of verificationism 'explicitation-verificationism'. (p. 24).

Com efeito, esta interpretação expressivista do verificacionismo parece se coadunar a vários tópicos do Wittgenstein intermediário onde ele parece apontar para o fato que a negação deve expressar ou tornar explícita a incompatibilidade presente no espaço de cores ou Satzsystem de cores. Wittgenstein afirma, por exemplo, que não vemos o vermelho, mas, por exemplo, que a azaleia é vermelha. Nesse sentido, vemos também que ela não é azul (WWK, 87).

Usando a distinção tradicional em filosofia da lógica entre inferência material e formal e o papel da negação no contexto do problema da exclusão de cores, podemos ver que Wittgenstein introduz o inferencialismo nas Satzsysteme pelo papel da negação e por dificuldades de expressão de inferências materiais com recursos estritamente formais. De fato, quando examinamos a volta de Wittgenstein à filosofia em 1929, podemos notar que: se existirem proposições elementares, elas todas deveriam estar inseridas em diferentes sistemas organizados conceitual e logicamente a partir de exclusões que a filosofia da lógica geralmente chama de materiais. As negações na linguagem deveriam ter o papel de explicitar muitas ou mesmo infinitas alternativas a proposição negada: "this point is not green, not red, not yellow, etc.".

As exclusões em Satzsysteme não são determinadas pelo significado dos conectivos lógicos, como em uma abordagem formal, mas sim pelas relações de incompatibilidade conceitual próprias destes espaços inferencialmente articulados. Em anotações de 25 de dezembro de 1929 em WWK, 
podemos examinar um mea culpa central em relação à visão de lógica baseada exclusivamente na noção formal de tautologia. Esta passagem é ilustrativa da tensão entre a demanda de análise exclusivamente verofuncional e a assunção de domínios holistas articulados inferencialmente:

Eu ainda não sabia de tudo isto na elaboração do meu trabalho [o Tractatus], e achava naquela época que toda dedução se baseava na forma da tautologia. Eu, naquela época, ainda não tinha visto que uma dedução também tem a forma: um homem tem $2 \mathrm{~m}$, então não tem $3 \mathrm{~m}$. Isto tem a ver com o fato de eu ter acreditado que as proposições elementares deveriam ser independentes, da existência de um estado de coisas não poder-se-ia deduzir a não-existência de um outro. Entretanto, se a minha perspectiva de hoje estiver correta, é até mesmo a regra que da existência de um estado de coisas pode-se deduzir a não-existência de todos os outros restantes que são descritos através do sistema de proposições. (p. 64, meu itálico).

Se a noção de tautologia não tivesse sido tão central no Tractatus, a evidente não-independência de complexos elementares organizados em sistemas, que deveria ser vista como regra em 1929, já poderia ter sido antecipada na época da elaboração do Tractatus. Note que o exemplo trazido por Wittgenstein na passagem acima, a saber, "se um homem tem $2 \mathrm{~m}$, então não tem 3m" é claramente um exemplo de exclusão baseada em relações conceituais que mapeiam incompatibilidades materiais e não em uma relação formal, tautológica.

É importante notar que este comportamento holista de proposições atômicas já estava no Tractatus, mas Wittgenstein não vira suas consequências adversas. Se ele tivesse prestado atenção em seus exemplos de cores no Tractatus teria notado isto, como afirma no revelador parágrafo 83 das PB. Nele, Wittgenstein assume o seu erro tractariano em um instrutivo mea culpa, ao articular a demanda de operadores lógicos, nesta altura, "sensíveis", i.e., de uso e escopo determinados e restritos pelo Satzsystem em que estamos operando, com limites de sua notação V-F (tabela de verdade) e a noção de sistema de coordenadas:

O conceito de proposição elementar perde agora totalmente o seu significado anterior. As regras sobre "e", "ou", "não" etc., que eu apresentei através da notação V-F, são apenas uma parte da gramática destas palavras, mas não toda ela. $\mathrm{O}$ conceito de coordenadas independentes na descrição: As proposições que, por exemplo, são ligadas através do "e" não são independentes uma da 
outra, mas elas formam uma figuração [Bild] e se deixam provar quanto à sua possibilidade ou impossibilidade de união. Na minha antiga visão de proposições elementares não havia a determinação de um valor de uma coordenada, apesar da minha observação que um corpo colorido está em um espaço de cores etc. teria me trazido diretamente para este ponto. (p. 111, meu itálico).

Para se operar e raciocinar com proposições nas Satzsysteme do retorno de Wittgenstein à filosofia, nós deveríamos conhecer o sistema em que a proposição está inserido, dominar suas incompatibilidades conceituais, para entendermos que combinações são permitidas e quais são proibidas. Concordo com Medina (2001) que a distinção entre inferências materiais e formais é fundamental neste contexto:

On this view, the interrelations that bring propositions together into systems are not formal inferential relations, but relations of material inference: inferential relations that are based on the content of the propositions involved. This inferentialist view constitutes a radical departure from the formalism of the Tractatus, according to which every inference is based on the logical form of the propositions involved and hence on content independent rules of logical syntax. (p. 305).

O Wittgenstein Intermediário defende ainda a ideia que entender o sentido de uma proposição significa entender o seu método de verificação. Pela contrapositiva, o princípio implicaria que falhar em entender o método de verificação de uma proposição significaria falhar em entender o sentido da proposição. De toda forma, este fato parece justificar, por exemplo, a insistência de Wittgenstein no entendimento de "eine Methode des Findens, Suchens" para se entender o que uma proposição quer dizer (PB 43). Por consequência, nós precisamos saber como determinar se algo é o caso para entender o sentido proposicional de uma expressão linguística. Note que a discussão do Wittgenstein intermediário não é sobre condições de verdade e como se referir a coisas, mas sobre sentido e como algum tipo de evidência a respeito de condições de asserção e consequências desta asserção pode restringir nosso entendimento de proposições.

O sentido de uma proposição é determinado pelo exame de como nós devemos proceder para determinar seu valor de verdade. Entender o sentido de uma proposição significa saber como o problema de sua verdade ou falsidade deve ser decidido (PB 243). Assim, o eixo temático de Witt- 
genstein gravita da visão de sentido como condições de verdade para a visão de sentido como condições de verificação ou como procedimentos de verificação. O significado do verificacionismo no período intermediário de Wittgenstein é permitir que exploremos a gramática, ou seja, as regras que governam o sentido das proposições, ao explicitarmos as regras pelas quais o sentido ou condições de verificação são determinadas. Estas regras explicitadas pelo método de verificação desempenham o papel de, por assim dizer, manter as proposições juntas em um sistema coerente e coeso, definindo uma maneira própria de falar sobre a realidade. (WWK p. 53; PB 216). Ao examinarmos o método peculiar de verificação de um Satzsystem, nós podemos identificar as regras deste sistema, uma vez que as regras do sistema definem a maneira em que as proposições podem ser comparadas com a realidade.

Neste contexto, é importante destacar que a imagem de verificacionismo obtida até agora já contraria a hipótese de Wrigley (1989) ou de Frascolla (2017) de que o Tractatus de Wittgenstein pressuporia (ou implicaria) verificacionismo. De fato, a distinção entre duas teorias semânticas, nomeadamente, uma baseada na semântica de condições de verdade e outra baseada em uma semântica fundada em condições para a atribuição de verdade, parece estar de fato no centro do retorno de Wittgenstein à Filosofia. Segundo Engelmann (2018), "the verification of a sentence, now, shows what we must understand in advance in order to compare it with reality" (p. 30). Como consequência, o sentido de uma proposição não deve pressupor sua própria verdade, mas somente elementos que torna possível a determinação desta verdade.

Seguindo Dummett $(1978,1991)$ e Kripke (1982), acredito que devemos ver o verificacionismo de Wittgenstein no começo da década de 1930 como uma ruptura com sua visão de significado no Tractatus e não assimilando o primeiro ao último. Wrigley (1989), por exemplo, parece não examinar suficientemente que o Tractatus mostra uma semântica de condições de verdade e que não há restrições baseadas em evidência ou método de obtenção concernentes à concepção de verdade. Como poderíamos dizer em filosofia da linguagem contemporânea, o Tractatus apresenta uma noção realista de conteúdo proposicional baseada em condições de verdade (TLP 
4.41, 4.431, 4.442, 4.45-4.461 e 4.463). Nele, o conteúdo proposicional deveria ser exaustiva e exclusivamente determinado por suas condições de verdade. Verificação ou prova deve ser irrelevante neste cenário tractariano. Como resultado, operadores tractarianos deveriam oferecer uma semântica realista neutra, ilimitada e essencialmente combinatória. Toda combinação de proposições elementares é sempre possível. O Tractatus é um excelente exemplo de uma abordagem na semântica baseada em leis da proposição, que não aceita restrições na formação de proposições moleculares de um conjunto de proposições atômicas legítimas.

No entanto, depois de alguns problemas, Wittgenstein nota que operadores lógicos deveriam ser topicamente sensíveis ao sistema que eles expressam (Prado Neto, 2003, Engelmann 2013, Cuter 2017). Segundo PB 8183, nós deveríamos testar ou verificar a possibilidade da formação molecular de algumas proposições, uma vez que a formação de algumas proposições complexas não seria permitida. Algumas construções deveriam ser proibidas. A livre distribuição de valores de verdade, independentemente da existência de nomes vazios, deveria ser restrita ou proibida em alguns sistemas organizados conceitualmente.

É possível relacionarmos o problema de Wittgenstein em expressar relações conceituais entre cores a alguns problemas com suas observações verificacionistas que emergem no contexto fenomenológico das suas Satzsysteme. Alguns exemplos destes Satzsysteme discutidos pelo Wittgenstein intermediário são sistemas de cores, temperatura, volume, dureza, comprimento, peso etc. Em todos estes sistemas fenomenológicos o princípio do terceiro excluído (PTE) parece não valer. A razão para esta restrição de validade do PTE é simples, como veremos na seção 3. Antes disto, discutiremos a impossibilidade de capturar pela negação tractariana o tipo de incompatibilidade que afligia Wittgenstein em seu retorno à Filosofia. Na próxima seção, investigaremos o comportamento lógico das sentenças que atribuem uma cor a um ponto do campo visual ou um grau à uma qualidade empírica. Elas são claramente mutuamente exclusivas, porque elas não podem ser verdadeiras juntas, mas elas não exibem o comportamento lógico da contradição, porque elas podem ser falsas juntas. Esta característica lógica identifica 
a incompatibilidade material no sistema de cores em particular e nos Satzsysteme de maneira geral.

\section{O problema da exclusão de cores como uma motivação para o inferencialismo}

Em 1923, Ramsey mostrou o primeiro grande desafio ao Tractatus, o assim chamado problema da exclusão das cores, em sua famosa resenha à primeira obra de Wittgenstein:

It is a principle of Mr. Wittgenstein's, and, if true, is a very important discovery, that every genuine proposition asserts something possible, but not necessary. This follows from his account of a proposition as the expression of agreement and disagreement with truth- possibilities of independent elementary propositions, so that the only necessity is that of tautology, the only impossibility that of contradiction. There is great difficulty in holding this; for Mr. Wittgenstein admits that a point in the visual field cannot be both red and blue; and, indeed, otherwise, since he thinks induction has no logical basis, we should have no reason for thinking that we may not come upon a visual point which is both red and blue. Hence he says that "This is both red and blue" is a contradiction. This implies that the apparently simple concepts red, blue (supposing us to mean by those words absolutely specific shades) are really complex and formally incompatible. He tries to show how this may be, by analysing them in terms of vibrations. But even supposing that the physicist thus provides an analysis of what we mean by "red" Mr. Wittgenstein is only reducing the difficulty to that of the necessary properties of space, time, and matter, or the ether. He explicitly makes it depend on the impossibility of a particle being in two places at the same time. These necessary properties of space and time are hardly capable of a further reduction of this kind. For example, considering between in point of time as regards my experiences; if $B$ is between $A$ and $D$ and $G$ between $B$ and $D$, then $G$ must be between $A$ and $D$; but it is hard to see how this can be a formal tautology (Ramsey, 1923/2012, p. 273).

Note que a dificuldade incontornável para o projeto tractariano da redução total de relações materiais entre conceitos a elementos puramente formais como a tautologia, como vimos, já é destacada pela objeção de Ramsey. Esta crítica motivou o exame do sistema de cores como primariamente um problema lógico e não como um problema epistêmico ou fenomenológico. A organização conceitual de um sistema de cores se impõe como um problema para a explicação tractariana da natureza da necessidade ser baseada somente em uma noção de tautologias formais. É neste contexto 
que no Tractatus “p. $\sim$ p” expressa a única forma de exclusão necessária. Entretanto, a passagem do Tractatus 6.3651 aponta que a conjunção "o ponto a do campo visual é azul" e "o mesmo ponto a é vermelho" deveria ser tomada como uma contradição. Como consequência, pode-se pensar qual é a natureza de uma proposição como "se a é verde, então não é vermelho", onde $a$ é um ponto do campo visual. Esta proposição parece fazer sentido e parece também ser necessária e verdadeira a priori. Contudo, como Ramsey (1923/2012) se perguntou, seria legítimo chamar esta proposição de tautologia? Ramsey parece defender uma visão tradicional formal da lógica no sentido de que não está preocupado com o conteúdo conceitual de proposições para determinar a validade de determinados raciocínios.

Em 1927, Ramsey retorna ao tópico da natureza da lógica e desenvolve o tema da exclusão de cores, ao discutir a natureza deste tipo de exclusão e as dificuldades com a explicação tractariana de necessidade, ele afirma:

it seems to me that formal logic is not concerned with it, but presupposes that all the truth-possibilities of atomic sentences are really possible, or at least treats them as being so. No one could say that the inference from 'this is red' to 'this is not blue' was formally guaranteed like the syllogism. (1927/2019, p.167).

Aqui Ramsey novamente apresenta a irredutibilidade lógica de elementos conceituais intrassistêmicos às tautologias formais tractarianas. A pressuposição de um horizonte combinatório sem restrições feito pela lógica formal, em se tratando de proposições elementares, é contrastado com a necessidade de restrições quando tratamos de inferências materiais, porque são baseadas em relações conceituais internas a um sistema inferencialmente organizado, como o de cores.

Com efeito, pode ser defendido que não há verdadeiramente um problema das cores no Tractatus. Que o problema da exclusão das cores não é um problema explícito para o Tractatus mostra como profundamente comprometido Wittgenstein estava com algumas importantes ideias logicistas em sua filosofia de juventude, como a demanda de uma análise completa e não-ambígua das proposições. É assumido na visão tractariana da linguagem que toda proposição com sentido deve ter uma forma lógica inequívoca que não pode ser vista pela superfície da linguagem. Neste sentido, a forma lógi- 
ca de uma proposição está escondida pela superfície gramatical da proposição e deveria ser invariavelmente muito complexa.

Com este cenário em mente, não é difícil entender por que no 6.3751 Wittgenstein argumenta que " $a$ é vermelho e a é azul" deveria ser uma instância de uma contradição, isto é, deveria exibir a forma lógica “p. $\sim p$ ”. Se nós só tivermos uma forma de necessidade lógica, a tautologia, então, pelo seu caráter dual, nós só teríamos uma forma de exclusão, a contradição. Isto parece que é o que está em discussão nas passagens tractarianas que começam na 6.37, nomeadamente que a única forma de impossibilidade lógica é a contradição formal "p. p p". Consequentemente, a surpreendentemente longa passagem 6.3751 desempenharia o papel de remover de uma vez por todas a possibilidade que a exclusão no sistema de cores representasse um contraexemplo possível para esta convicção filosófica.

Note que este problema não precisa ser pensado como restrito ao sistema de cores, uma vez que pode ser visto em outros sistemas conceitualmente organizados. Considere as seguintes sentenças:

i. A mesa ali na frente tem três metros de comprimento e (a mesma) mesa ali na frente tem quatro metros de comprimento.

ii. Agora são 25 graus Celsius no Recife e agora são 26 graus Celsius no Recife.

iii. Flamengo perdeu ontem e Flamengo ganhou ontem.

iv. O animal ali é um gato e (o mesmo) animal ali é um cachorro.

A peculiaridade destes exemplos, que parecem compreender o mesmo tipo de exclusão material presente no sistema de cores, é a de que nenhuma negação formal explícita é encontrada neles. Além disso, se a conjunção expressa em todos estes exemplos fosse de fato contraditória, a disjunção correspondente deveria ser uma tautologia. Contudo, nenhum dos seguintes exemplos são tautologias: "o ponto a no campo visual é azul ou vermelho", "a mesa ali adiante tem três metros ou (a mesma) mesa tem quatro metros de comprimento", "o animal ali é um gato ou é um cachorro", “meu time de futebol ganhou ou perdeu ontem". A sentença "agora são 25 graus ou 26 graus no Recife" é tampouco uma tautologia. 
É crucial notar que o padrão lógico que estes sistemas conceituais compartilham é o de contrariedades, como mostrado no tipo usual de relações conceituais em sistemas taxonômicos e em algumas regras. Note também que a analogia feita por Wittgenstein em SRLF (1929) a respeito da tentativa de pôr Jones e Brown juntos em uma cadeira onde só caberia uma pessoa espelha perfeitamente o problema da exclusão baseada em um padrão lógico distinto do da contradição (p. 169).

Neste exemplo, as proposições não podem ser verdadeiras juntas, mas podem muito bem ser falsas juntas, uma vez que a cadeira pode ser ocupada por qualquer outra pessoa. Analogamente, "o ponto a do campo visual é vermelho" e "o ponto a do campo visual é azul" não podem ser verdadeiras juntas, mas podem ser falsas juntas, por não esgotarem as alternativas. Com efeito, o ponto poderia ter qualquer outra cor além de vermelho ou azul. Neste contexto, parece-nos útil tentar capturar o comportamento lógico das contrariedades com a notação tractariana. A motivação para esta estratégia surge das discussões em 1929 e dos trabalhos de Von Wright (especialmente, 1996/2011) em lógicas modais. O grande fio condutor é o seguinte: se a organização lógica presente nos conceitos de cores representa um problema para a lógica tractariana, deveria representar um problema para sua notação também. ${ }^{3}$ Ampliamos a tese expressivista para mostrar que algumas mutilações das tabelas de verdade deveriam explicitar relações inferências nos Satzsysteme. Aqui nós examinamos a distinção entre contradição e contrariedade, usando tabelas de verdade, para comparar as tabelas de verdade em 1921 e em 1929.

Em 1921, nós temos a seguinte tabela de verdade para a conjunção:

\begin{tabular}{|c|c|c|}
\hline $\mathbf{p}$ & $\mathbf{q}$ & $\mathbf{p} \cdot \mathbf{q}$ \\
\hline $\mathrm{V}$ & $\mathrm{V}$ & $\mathrm{V}$ \\
\hline $\mathrm{V}$ & $\mathrm{F}$ & $\mathrm{F}$ \\
\hline $\mathrm{F}$ & $\mathrm{V}$ & $\mathrm{F}$ \\
\hline $\mathrm{F}$ & $\mathrm{F}$ & $\mathrm{F}$ \\
\hline
\end{tabular}

\footnotetext{
${ }_{3}^{3}$ Para uma discussão a respeito do desenvolvimento de elementos notacionais relacionados à linguagem fenomenológica no Wittgenstein intermediário, ver Engelmann 2017. Para uma investigação a respeito de Wittgenstein poder ser considerado como pai das tabelas de verdade, ver Silva 2016c.
} 
A tabela de verdade acima pode ser interpretada da seguinte maneira pouco problemática usando linguagem natural:

\begin{tabular}{|c|c|c|}
\hline João é cientista & João é logico & João é cientista e João é lógico \\
\hline V & V & V \\
\hline V & F & F \\
\hline F & V & F \\
\hline F & F & F \\
\hline
\end{tabular}

Ao aplicarmos a "solução" de 6.3751 para o problema da exclusão de cores ao mesmo tipo de tabela de verdade, nós teríamos alguma tabela como a seguinte, onde a última coluna mostra a conjunção como uma contradição:

\begin{tabular}{|c|c|c|}
\hline A é vermelho & A é azul & A é vermelho e A é azul \\
\hline V & V & F \\
\hline V & F & F \\
\hline F & V & F \\
\hline F & F & F \\
\hline
\end{tabular}

Note que a tabela acima não mostra a negação requerida para tornar a conjunção uma contradição. A resposta do Tractatus seria que nós deveríamos continuar analisando a proposição para, no fim, descobrir a contradição. Contudo, o Tractatus não diz em nenhum lugar se "a é vermelho" é uma proposição atômica ou não. Como vimos, este problema está em perfeito acordo como a maneira lógico-atomista de investigar problemas filosóficos em geral e da linguagem em particular: proposições complexas devem ter uma única forma lógica (aqui, a contradição), que está escondida (não visível na superfície da gramatica), é muito complexa e deve ser descoberta pelo filósofo(-lógico).

Este problema ainda está saliente quando Wittgenstein volta à filosofia, como podemos ver na maneira como ele retorna a representações diagramáticas de exclusões, tais como as presentes em sistemas de cores, em seu Nachlass. Por exemplo, ele retorna às tabelas de verdade no ensaio de 1929. O que está acontecendo em 1929 não é tecnicamente muito significativo, 
mas é filosoficamente importante. Em 1929, Wittgenstein mantém a ideia Russelliana de análise completa, mas começa a falar da necessidade de adição de regras. Neste contexto, o problema com última tabela de verdade não seria com o valor de verdade de sua última coluna. Note que a exclusão ela mesma não é absurda, mas sim a sua representação diagramática ao usarmos a tabela de verdade completa que é absurda.

O problema das cores mostra que o autor do Tractatus tem de expandir o seu vocabulário de expressões sinnlose, baseado antes exclusivamente em tautologias e contradições; o vocabulário lógico antigo era limitado. Em um sentido, há o reconhecimento que existem mais Sinnlose Sätze que tautologias e contradições. O problema da exclusão de cores parece desafiar a ideia contida em 3.325 a respeito da identidade da gramatica lógica e da sintaxe, se a sintaxe lógica for considerada exaustivamentente como tautologias e contradições. Em nossa visão, a novidade na concepção de Wittgenstein é que a gramática deveria incluir mais do que tautologias e contradições. Grammatik deveria também incluir o que convencionalmente é chamado na tradição filosófica de inferências materiais tais quais como codificadas no sistema de cores ou de comprimento.

Em 1929, o problema está com o esquema ele mesmo, com a livre distribuição de valores de verdade. Wittgenstein percebeu que o procedimento combinatório na semântica tractariana deveria seguir regras adicionais, porque os operadores deveriam começar a ser sensíveis à organização conceitual de cada sistema. Como nós vemos no seguinte esquema da tabela de verdade:

\begin{tabular}{|c|c|}
\hline $\begin{array}{l}\text { A é vermelho } \\
\text { A tem 3 metros de comprimento } \\
\text { Agora são 28 graus Celsius }\end{array}$ & $\begin{array}{l}\text { A é azul } \\
\text { A tem 4 metros de comprimento } \\
\text { Agora são 29 graus Celsius }\end{array}$ \\
\hline V & F \\
\hline F & V \\
\hline F & F \\
\hline
\end{tabular}

Uma linha deve ser retirada, removida, bloqueada, ou em uma expressão mais dramática "mutilada" em diferentes Satzsysteme (Von Wright, 1996/2011). Algumas combinações devem ser bloqueadas ad hoc, isto é, de- 
pendendo com qual sistema estejamos operando. Impor restrições à formação de tabelas de verdade significa impor restrições em leis das proposições, como vero-funcionalidade, extensionalidade e a outras características típicas do ambiente clássico do Tractatus. Isto significa uma virada filosófica dramática. Nós temos que adicionar regras para restringir o espaço lógico. ${ }^{4}$

Em WWK, Wittgenstein sugere que o espaço lógico deve ser "eingeengt" (limitado, restrito) por regras especiais para a sintaxe interna das proposições elementares que proíbem algumas combinações (p. 80). O que não é proibido por estas regras é permitido. Em outros casos, vero funcionalidade mantém seu poder. Intuitivamente, não faz sentido falar de regras quando todas as combinações e possibilidades são autorizadas. Uma regra significa entre outras coisas restringir um espaço de possibilidades, de atividades, de ações, um Spielraum. Em Wiener Ausgabe I, nós vemos, por exemplo, a discussão sobre a mutilação de uma linha de uma tabela de verdade clássica para a conjunção que coincide com a expressão do paradigma da contrariedade. ${ }^{5}$ Nestas passagens, Wittgenstein escreve sobre o "Wegfall der ersten Linie" ["remoção da primeira linha"] e também "eine Reihe einfach durchstreichen. Ich muss die ganze ober Reihe durchstreichen" [simplesmente riscar uma fileira, i.e., considerá-la impossível. Eu tenho que riscar a linha superior inteira] (p. 58), "Die ganze ausstreichen Linie" ["riscar a linha inteira"] (p. 59) e "die obere Linie streichen" ["eliminar a linha superior"] (p. 59). Esta discussão da mutilação da tabela de verdade corresponde a discussões bastante relevantes em WWK (pp. 64 e 92) e PB 81.

De acordo, podemos aumentar o poder expressivo de tabelas de verdade. As mutilações sistemáticas da tabela de verdade expressam alguns importantes padrões conceituais e lógicos, tais como:

1. A contrariedade - uma vez que duas proposições não podem ser ambas verdadeiras, mas podem ser falsas juntas, removemos a primeira combinação VV e mantemos a última combinação FF:$$
\begin{array}{|l|l|}
\hline \mathbf{p} & \mathbf{q} \\
\hline
\end{array}
$$

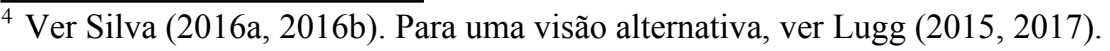

${ }^{5}$ Ver Silva (2017) para uma discussão detalhada do reconhecimento do Wittgenstein Intermediário da relevância da distinção entre contrariedade e contradição no contexto do assim chamado problema da exclusão de cores.
} 


\begin{tabular}{|c|c|}
\hline $\mathrm{V}$ & $\mathrm{F}$ \\
\hline $\mathrm{F}$ & $\mathrm{V}$ \\
\hline $\mathrm{F}$ & $\mathrm{F}$ \\
\hline
\end{tabular}

2. A subcontrariedade - uma vez que duas proposições não podem ser ambas falsas juntas, mas podem ser verdadeiras juntas, removemos a última combinação FF e mantemos a primeira combinação VV:

\begin{tabular}{|c|c|}
\hline $\mathbf{p}$ & $\mathbf{q}$ \\
\hline $\mathrm{V}$ & $\mathrm{V}$ \\
\hline $\mathrm{V}$ & $\mathrm{F}$ \\
\hline $\mathrm{F}$ & $\mathrm{V}$ \\
\hline
\end{tabular}

3. A contradição - uma vez que duas proposições podem nem ser verdadeiras juntas e nem falsas juntas, removemos a primeira combinação TT e a última combinação FF:

\begin{tabular}{|c|c|}
\hline $\mathbf{p}$ & $\mathbf{q}$ \\
\hline $\mathrm{V}$ & $\mathrm{F}$ \\
\hline $\mathrm{F}$ & $\mathrm{V}$ \\
\hline
\end{tabular}

\section{Alguns problemas com o princípio do terceiro excluído (PTE)}

Nós vimos como a negação no Satzsysteme de Wittgenstein intermediário deveria expressar diferentes relações conceituais de incompatibilidade em padrões lógicos relacionados com contrariedades e não com contradições. Estas duas relações de contradição e de contrariedade entre pares de proposições significativas são centrais para a nossa discussão da relevância de cores para a guinada inferencialista na filosofia do Wittgenstein intermediário, porque desloca uma tradição de semântica fundada no atomismo lógico e referencialismo para um inferencialismo e holismo semânticos para a determinação do significado de nossas expressões linguísticas (Brandom 2008; Peregrin e Svoboda, 2017; Stekeler-Weithofer, 2013). 
Com esta distinção em mente, nós podemos reconsiderar casos de inconsistência ou de absurdidade, separando-as em duas categorias distintas e irredutíveis comumente usadas confusamente na discussão do assim chamado problema das cores. Nós podemos caracterizar a primeira forma de exclusão pela contradição desta forma: duas proposições se contradizem ou são opostas por contradição se e somente se elas não poderem nem ser verdadeiras juntas e nem falsas juntas. Em outras palavras, quando uma proposição é falsa, a outra deve ser necessariamente verdadeira e vice-versa. Esta é uma explicação razoável para o requerimento de "p. p p" ser sempre falsa, nomeadamente, o valor de verdade de "p", qualquer que seja, é o oposto contraditório de valor de verdade de " $\sim \mathrm{p}$ ".

De um modo complementar, nós podemos caracterizar a segunda forma da exclusão relevante para nossa discussão, nomeadamente a exclusão por contrariedade, do seguinte modo: duas proposições se opõem por contrariedade, ou um par de proposições excluem uma a outra por contrariedade, se estas proposições não puderem ser verdadeiras juntas. Como no caso da contradição, o fato que as proposições não possam ser verdadeiras juntas caracteriza a inconsistência ou incompatibilidade dentro do par. Entretanto, no caso da contrariedade, as proposições em oposição, podem, em contraste com o comportamento lógico da contradição, ser falsas juntas.

De acordo, Brandom, concernente à noção de incompatibilidade material que usa em seu inferencialismo semântico, vê a necessidade de pensar uma negação, diferente da negação clássica, que possa expressar a contrariedade própria nestes domínios onde o conteúdo de uma proposição é determinado a partir do conjunto de proposições que são incompatíveis com ela.

...can be thought of as a sort of conceptual vector-product of a negative component and a modal component. It is compossibility. To use this semantic notion to introduce a negation operator into the object vocabulary, we must somehow isolate and express explicitly that negative component. The general semantic model we are working with represents the content expressed by a sentence by the set of sets of sentences incompatible with it. So what we are looking for is a way of computing what is incompatible with negated sentences (and, more generally, with sets of sentences containing them). Since we do not have any sort of yes/no evaluation of sentences in the picture (not even a relativized one), we cannot approach negation as a kind of reversal of semantic 
polarity. How else might we think about it? Incompatible sentences are Aristotelian contraries. (Brandom, 2008, p. 126).

Instrutivamente, exemplos paradigmáticos para estes tipos de inconsistência baseado na contrariedade aristotélica, como Brandom aponta, pode ser achada em todos os sistemas já apresentados nos et ceteras do Tractatus 2.0131 e nos et ceteras de SRLF (pp. 165 e 167). No ensaio de 1929, Wittgenstein parece reconhecer algo que ele não previra no Tractatus: a necessidade de desenvolver uma fenomenologia de domínios conceitualmente organizados (Satzssysteme). Esta fenomenologia peculiar, como um projeto a ser buscado neste estágio na filosofia de Wittgenstein, é fortemente sugerida no último parágrafo de SRLF (Engelmann, 2013, 2014, 2018)

Retornemos à primeira pergunta a respeito de verificacionismo na volta de Wittgenstein à Filosofia. Alguns autores conectam o Wittgenstein intermediário com alguma forma de construtivismo em função de Brouwer e/ou da influência do Círculo de Viena (Marion, 1998), ou mesmo tentam encontrar já no Tractatus algumas ideias construtivistas (Wrigley, 1989, Newen, 1997/2015), como procedimentos e operações sendo importantes para a formação do sentido proposicional. Entretanto, é importante notar que os Satzsysteme de Wittgenstein são inferencialmente densos, ou seja, a negação de uma proposição torna explícito quadros de incompatibilidade material e conexões conceituais com muitas outras alternativas. Como resultado nos Satzsysteme fenomenológicos de Wittgenstein (como cores, temperatura, volume, dureza, comprimento, peso, altura etc.), nós deveríamos reconhecer que o princípio do terceiro excluído não vale, como em muitas escolas construtivistas (Marion, 1998). A validade de PTE deveria ser restrita. A razão para esta restrição construtiva é simples: o terceiro termo não é excluído em nenhum Satzsystem.

De acordo, Horn e Wansing (2015) defendem que, embora opostos contraditórios sejam mutuamente exaustivos e exclusivos, opostos contrários não exaurem as alternativas do seu domínio conceitual:

As introduced in Aristotle's Categories (11b17), the genus of opposition (apophasis) is divided into species that include contrariety and contradiction. Contradictory opposites, whether affirmative and negative counterparts of a singular predication (Socrates is wise/Socrates isn't wise) or quantified expressions (All 
pleasure is good/Some pleasure is not good), are mutually exhaustive as well as mutually exclusive, while contrary opposites (Socrates is wise/Socrates is unwise; All pleasure is good/No pleasure is good) do not mutually exhaust their domain. Contraries cannot be simultaneously true, though they may be simultaneously false. Members of a contradictory pair cannot be true or false simultaneously; contradictories "divide the true and the false between them". Contrary terms (enantia) come in two varieties (Cat. 11b38ff.). In immediate or logical contraries (odd/even, sick/ well), a true middle - an entity satisfying the range of the two opposed terms but falling under neither of them-is excluded, e.g., an integer neither odd nor even. But mediate contrary pairs (black/ white, good/bad) allow for a middle - a shirt between black and white, a man or an act neither good nor bad. Neither mediate nor immediate contraries fall under the Law of Excluded Middle [LEM] (tertium non datur).

É importante notar que pares contrários permitem uma terceira alternativa, ou seja, apesar de serem incompatíveis entre si, eles não esgotam as alternativas conceituais previstas pelo sistema onde estão inseridos. Em outras, palavras para todo $\mathrm{p}$ e q, se eles forem opostos, $\mathrm{p}$ e $\mathrm{q}$ não podem ser verdadeiros juntos. Contudo, se a oposição é contraditória, ou p ou q deve ser verdadeiro. Entretanto, se "a é azul”, por exemplo, de fato nega ou exclue "a é vermelho", então nós devemos aceitar que ambos podem ser falsos juntos. Em outras palavras, é possível que nenhum dois seja de fato verdadeiro. Como no caso de Brown e Jones, no exemplo da SRLF, poderia ser o caso de nenhum dos dois ocupar a cadeira de um só lugar. Ou como no caso da medição de uma mesa, ela não pode ter 3 metros e quatro metros simultaneamente, embora ela possa nem ter 3 metros e nem 4 metros de comprimento.

Em atribuições de cores a pontos do campo visual, ou da atribuição de um grau a uma propriedade empírica, a incompatibilidade por contrariedade é o padrão lógico a ser expresso pela negação. Neste contexto, nós temos que lidar com uma indeterminação conceitual ou vagueza obrigatórias. Por exemplo, muito pouco é sabido a respeito da cor de uma camisa ou do comprimento de uma mesa se alguém disser, por exemplo, "minha camisa não é vermelha" ou "a mesa não tem 3 metros de comprimento." Se "a é vermelho" for $\mathrm{p}$, então $\sim \mathrm{p}$ não precisa ser “ $a$ é azul”, uma vez que a pode ter todas as outras cores, um número infinito de outras cores, se nós permitirmos que o sistema de cores seja denso, ou seja, se permitirmos que para 
cada duas cores sempre haja uma outra entre as duas. Note que não faz sentido se falar de "o contrário" de uma proposição, uma vez que esta pode ter vários ou infinitos contrários, embora possamos falar de "o contraditório" de uma proposição dada.

Em todo caso, o significado de "vermelho" pode ser dado ao apontarmos para uma amostra vermelha, mas não por dizer "não" ou gesticular de maneira desaprovadora enquanto apontando para uma amostra azul. Em nenhum dicionário há um " $\mathrm{x}$ " sobre uma amostra azul para definirmos o que é vermelho. No paradigma da contrariedade, nós temos um importante tipo de assimetria, porque a proposição afirmativa parece dizer mais que a negativa, uma vez que a negação expressiva de incompatibilidades materiais em sistemas organizados conceitualmente indetermina coisas. A proposição com a negação que expressa contrariedades explicita indeterminações no quadro de exclusões dos Satzsysteme.

Como nós vimos, a ideia de uma negação formal clássica, como a que expressa a contraditoriedade, é determinada pela simetria em introduzir não-A como a contraditória de A. Nestes casos, a relação da contradição pode ser definida em termos de certas leis lógicas, tais como a lei do terceiro excluído e a lei da não-contradição. Entretanto, a negação nos Satzsysteme não pode ser pensada como um mero comutador de condições de verdade em função das assimetrias conceituais nestes sistemas. Como nós vimos, mesmo que a conjunção de " $a$ é vermelho" e "a é azul" pudesse ser tomada como uma contradição, a disjunção de ambos não é uma tautologia, independente do uso de uma disjunção exclusiva ou inclusiva.

\section{Conclusão}

Faz sentido defender que o Tractatus mostra um tipo de semântica realista do sentido proposicional, porque o significado de uma proposição é única e exclusivamente determinado por suas condições de verdade. Isto é capturado ao introduzirmos operadores lógicos via tabelas de verdade. Entretanto, quando Wittgenstein retorna à Filosofia, depois do reconhecimento dos pontos sem saída de demandar que toda necessidade é tautológica, algumas mutilações das tabelas de verdade deveriam explicitar relações inferen- 
ciais nos Satzsysteme. Em WWK, por exemplo, Wittgenstein afirma que “Tautologie ist gana nebensächlich" (p. 91).

Isto mostra a razão pela qual o Wittgenstein Intermediário surpreendentemente advoga abordagens mais construtivas para a formação do sentido proposicional. Em seu verificacionismo, o Wittgenstein Intermediário afirma, dentre outras coisas, que entender o significado de proposições seria entender seu método de verificação (PB pp. 174, 200) e WWK $(47,53,79)$. Em outras palavras, se $\mathrm{p}$ tem sentido, nós deveríamos saber como verificála. Desta perspectiva, não saber como verificar p significa não entender o significado de p ou mesmo duvidar se p tenha algum sentido. Em semânticas realistas, não há restrições construtivas para a formação de proposições, e nós podemos sempre, em princípio, atribuir um valor de verdade independente de sua complexidade ou da forma de verificá-la, ou mesmo se não for possível verificá-la ou não sabermos como verificá-la. Esta visão tractariana deve naturalmente passar por mudanças, uma vez que o processo de formação de proposições complexas não pode ser feito ou garantido sem a determinação de quando e em quais domínios algumas combinações são autorizadas e outras proibidas.

Vimos como o verificacionismo de Wittgenstein demanda que se nós tenhamos que restringir a formação de proposições complexas, é crucial testar, verificar se uma proposição complexa é possível ou não, em outras palavras, se a proposição complexa é permitida ou não. Isto é feito ao explicitarmos as regras que permitem a formação do sentido proposicional. Se a formação de proposições elementares não é autorizada, digamos, pelas regras fenomenológicas (de cores, comprimento, volume, temperatura, dureza, etc.), a atribuição ou determinação de valores de verdade deveria ser restrita em cada circunstância de aplicação. É importante notar que em 1929, Wittgenstein se engajou em uma forma peculiar de fenomenologia, onde o autor manteve a ideia tractariana de análise completa, mas rejeitou a independência de proposições elementares (WWK 73-74).

Neste sentido, novas regras deveriam ser introduzidas para restringir combinações de proposições elementares; elas não poderiam mais ser fundadas na semântica de condições de verdade baseadas na bipolaridade tractariana. Isto marca o tipo de inferencialismo que aparece no Wittgenstein 
Intermediário. Se nós tivermos proposições elementares, elas deveriam todas estar inseridas em diferentes sistemas que são organizados pelas exclusões por contrariedade e não por contradição onde a negação explicita a existência de várias alternativas nestes sistemas inferencialmente organizados. Para operar com proposições deveríamos saber o sistema onde a proposição está inserida para checar quais combinações são permitidas e quais são proibidas. Como resultado, nós devemos ao menos ter dois tipos de modalidade para lidar com esta semântica de incompatibilidades por contrariedade, uma modalidade combinatória e uma fenomenológica ou material. A exclusão de cores é uma boa motivação para inverter as prioridades tradicionais. Primeiro, a modalidade epistêmica deveria ser entendida em termos de articulações inferenciais que fixam o conteúdo conceitual e que ferem o PTE e depois procedimentos combinatórios ou formais poderiam ser introduzidos para explorar relações mais abstratas e clássicas.

\section{Referências bibliográficas}

AYER, A. J. (1995). Language Truth \& Logic. $2^{\text {nd }}$ edition. Dover Publications (1st ed.936).

BLACK, M. (1986). "Verificationism and Wittgenstein's Reflections on

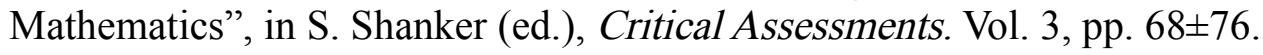

BRANDOM, R (2008). Between Saying and Doing: Towards an Analytic Pragmatism. Oxford: Oxford University Press. DOI: 10.1093/acprof:oso/9780199542871.001.0001

CARNAP, R. (1991). Autobiography in The Philosophy of Rudolf Carnap, Schilpp, P. A. (ed.). (La Salle, IL: Open Court, 1991).

COFFA, J. A. (1991). The Semantic Tradition from Kant to Carnap, Cambridge University Press, Cambridge.

CUTER, J. V. (2017). Uma fenomenologia sem métrica. In: Fenomenologia, análise e gramática: comentário às observações filosóficas de Wittgenstein parte 1. Sao Paulo: Ed. Mundaréu.

ENGELMANN, M. L. (2013). Wittgenstein's philosophical development: Phenomenology, Grammar, Method, and the Anthropological View. London: Palgrave. 
ENGELMANN, M. L. (2014). “Verificacionismo e 'Mito do Dado' no Contexto das Observações Filosóficas." Analytica 18(2): 13-40.

ENGELMANN, M. L. (2017). "What Does a Phenomenological Language Do? (Revisiting Some Remarks on Logical Form in its Context)." In Colors in the Development of Wittgenstein's Philosophy. Edited by M. Silva. Cham: Palgrave Macmillan.

ENGELMANN, M. L. (2018). Phenomenology in Grammar: ExplicitationVerificationism, Arbitrariness, and the Vienna Circle. In Kuusela, Ometita, Uçan (ed.). Wittgenstein and Phenomenology. New York: Routledge.

DUMMETT, M. (1978). 'The Significance of Quine's Indeterminacy Thesis', in Truth and Other Enigmas. Cambridge, MA.: Harvard University Press.

DUMMETT, M. (1991). The Logical Basis of Metaphysics. Cambridge MA.: Harvard University Press.

FRASCOLLA, P. (2017). "Ontology and semantics: an anti-realistic reading of the Tractatus". Belgrade Philosophical Annual 30: pp. 139-149. DOI: 10.5937/BPA1730139F

HACKER, P. M. S. (1986). Insight and Illusion. Themes in the Philosophy of Wittgenstein. Revised edition. Oxford: Clarendon Press.

HORN, L. R.; WANSING, H. (2015). "Negation". In: The Stanford Encyclopedia of Philosophy (Spring 2017 Edition), ed. Edward N. Zalta. Stanford CA: Stanford University. Consulted on October $4^{\text {th }}, 2018$. Available at https://plato.stanford.edu/archives/spr2017/entries/negation/.

KRIPKE, S. (1982). Wittgenstein on Rules and Private Language. Oxford: Blackwell.

MEDINA, J. (2001). Verificationism and Inferentialism in Wittgenstein's Philosophy. Philosophical Investigations 4:4: 304-33.

LUGG, A. (2015). "Wittgenstein on Colour Exclusion: Not fatally mistaken." Grazer Philosophische Studien 92, no. 1: pp. 1-21. DOI: 10.1163/9789004310841_002

LUGG, A. (2017). "Incompatible Colours and the Development of Wittgenstein's Philosophy". In: Colours in the development of Wittgenstein's Philosophy, edited by M. Silva. London: Palgrave Macmillan. pp. 33-55. DOI: 10.1007/978-3-319-56919-2_3

MARION, M. (1998). Wittgenstein, finitism, and the foundations of mathematics. Oxford: Oxford University Press. 
NEWEN, A. (1997). "Die Entwicklung der Wittgensteinischen Sprachphilosophie von 1929-1932". Zeitschrift für philosophische Forschung 5, no. 3: pp. 427-447. Traduzido ao português por Marcos Silva. Princípios: Revista de Filosofia, Natal, v. 22, n. 39, set.-dez. 2015. ISSN1983-2109. http://www.periodicos.ufrn.br/principios/article/view/6535

PEREGRIN, J.; SVOBODA, V. (2017). Reflective Equilibrium and the Principles of Logical Analysis Understanding the Laws of Logic. New York: Routledge.

PRADO NETO, B. (2003). Fenomenologia em Wittgenstein. Tempo, Cor e Figuração. Rio de Janeiro: Editora UFRJ.

RAMSEY, F. (1923). “Tractatus Logico-Philosophicus. By Ludwig Wittgenstein, with an Introduction by Bertrand Russell. (International Library of Psychology, Philosophy and Scientific Method.) London. Kegan Paul, Trench, Trubner \& Co. Ltd., 1922. Pp. 189. 10s. 6d.". Mind, Vol. 32, No. 128: pp. 465-478. DOI: 10.1093/mind/XXXII.128.465 Traduzida ao português por Marcos Silva. PHILÓSOPHOS, GOIÂNIA, V.17, N. 2, P. 263-288, JUL./DEZ. 2012.

RAMSEY, F. (1927). "VI.-Symposium: 'Facts and Propositions' I". Proceedings of the Aristotelian Society, Supplementary Volumes, Vol. 7, No. 1, Mind, Objectivity and Fact: pp. 153-206. DOI: 10.1093/aristoteliansupp/ 7.1.153. Traduzida ao português por Marcos Silva. PHILÓSOPHOS, GOIÂNIA, V. 24, N. 1, P. 347-367, JAN./JUN. 2019.

SILVA, M. (2016a). "Two forms of exclusion mean two different negations". Philosophical Investigations 39, no. 3: pp. 215-236. DOI: 10.1111/phin. 12068

SILVA, M. (2016b). "On a Philosophical Motivation for Mutilating Truth Tables". Nordic Wittgenstein Review 5, no. 1: pp. 105-126.

SILVA, M. (2016c). "Could Wittgenstein still be held as a father of truth tables?" O que nos faz pensar 25, no. 39: pp. 123-146.

SILVA, M. (2017). "Wittgenstein on contradiction and contrariety: Four turning points in the development of his philosophy of logic". In: Colours in the development of Wittgenstein's Philosophy, edited by M. Silva. pp. 185214. London: Palgrave.

STEKELER-WEITHOFER, P. (2013). "Regula et sententia convertuntur: On classified inferences in linguistic understanding". Argumentos 5, no. 10: pp. $72-96$.

VON WRIGHT, G. H. (1996). "On Colour: A Logico- Philosophical Fantasy". In Six Essays in Philosophical Logic. Helsinki: Acta Philosophica Fennica. pp. 9-16. Traduzido ao português por Marcos Silva. ANALYTICA, Rio de Janeiro, vol $15 \mathrm{n}^{\circ}$ 2, 2011, p. 265-275. 
WITTGENSTEIN, L. (1931/1984). Philosophische Bemerkungen [PB]. Werkausgabe Band 2. Frankfurt am Main: Suhrkamp.

WITTGENSTEIN, L. (1984). Tractatus Logico-philosophicus [TLP]. Tagebücher 1914-16. Philosophische Untersuchungen. Werkausgabe Band 1. Frankfurt am Main: Suhrkamp.

WITTGENSTEIN, L. (1929). "Some Remarks on Logical Form" [SRLF]. Proceedings of the Aristotelian Society, Supplementary Volumes, Vol. 9, Knowledge, Experience and Realism, pp. 162-171 Published by: Blackwell Publishing on behalf of The Aristotelian Society.

WITTGENSTEIN, L. (1984). Wittgenstein und der Wiener Kreis (19291932) [WKK]. Werkausgabe Band 3. Frankfurt am Main: Suhrkamp.

WITTGENSTEIN, L. (2000). Wittgenstein's Nachlass. The Bergen Electronic Edition. Oxford.

WITTGENSTEIN, L. (2000). Wiener Ausgabe I. Ed. M. Nedo. Springer.

WITTGENSTEIN, L. (1979b). Wittgenstein's Lectures, Cambridge $1932 \pm 35$, from the notes of A. Ambrose and M. MacDonald, edited by A. Ambrose, Blackwell, Oxford.

WRIGLEY, M. (1989). The Origins of Wittgenstein's Verificationism. Synthese 7: 65-90. 